\title{
Percepción de riesgo asociada a la prescripción continuada de benzodiacepinas en salud mental y atención primaria
}

\author{
Risk perception in the ongoing prescription of \\ benzodiazepines in mental health and primary care
}

\author{
Hugo López Pelayo****; Marina Fàbrega Ribera*; Albert Batalla Cases****; Juan Miguel \\ Garrido Ocaña**; María Mercedes Balcells Oliveró***; Antoni Gual Solé*** \\ *Servicio de Psiquiatría. Institut Clínic de Neurociències. Hospital Clínic i Universitari de Barcelona. **Centre Salut Mental \\ Esquerra de la Eixample. Servicio de Psiquiatría. Institut Clínic de Neurociències. Hospital Clínic i Universitari de Barcelona. \\ ***Unitat de Conductes Addictives. Hospital Clínic i Universitari de Barcelona. ****Fundació Clínic Recerca Biomèdica.
}

$\mathrm{E}$ 1 grupo farmacológico de las benzodiacepinas tiene múltiples indicaciones que se traducen en una amplia utilidad clínica a corto plazo. En los últimos años se ha objetivado un incremento en el uso de benzodiacepinas, llegando a ser el aumento del $56 \%$ desde 1995 hasta 2002 (García del Pozo et al., 2004). El consumo continuado (3 meses o más) de hipnosedantes es de hasta $2.7 \%$ de la población adulta (15-64 años) (Plan Nacional sobre Drogas, 2009). Pese a que la evidencia sobre su eficacia en la prescripción crónica es escasa y los riesgos asociados a su uso prolongado son notables, su utilización crónica es frecuente. En el año 2000, la Agencia Española del Medicamento ya advertía que la prescripción debía ser lo más corta posible, reevaluable regularmente -sobretodo en los pacientes libres de síntomas-, y que la duración del tratamiento no debería sobrepasar las 8-12 semanas incluyendo la retirada gradual.

Los prescriptores tienen un papel importante en este problema de salud pública, pues a diferencia de otras sustancias de abuso, el acceso puede ser bajo prescripción médica. La percepción de riesgo de prescripción a largo plazo del médico es un punto clave. El concepto de percepción del riesgo es subjetivo y multidimensional. Algunos autores proponen diversas dimensiones de la percepción de riesgo que pretenden ser descriptivas y poco explicativas (Slovic, 1987) intentado minimizar la subjetividad de la percepción (Puy Rodríguez, 1994).

Hemos estudiado 26 dimensiones de la percepción de riesgo de la prescripción continuada de benzodiazepinas (tabla 1) en 12 profesionales de atención primaria (AP) y 25 de salud mental (SM) mediante una encuesta autoadministrada, con una escala Likert ( 1 a 7). Se consideró percepción escasa 1 punto, percepción baja 2 puntos, percepción media-baja 3 puntos, percepción media 4 puntos, percepción media-alta 5 puntos, percepción elevada 6 puntos y percepción muy elevada 7 puntos. Se usó la prueba paramétrica t de student para muestras independientes para comparar las medias de variables continuas. La relación entre variables continuas se estudió mediante correlaciones de Pearson.

De los 37 especialistas en psiquiatría que se les solicitó la participación respondieron $25(67,6 \%)$. De los 22 médicos de familia que se les solicitó la participación respondieron $12(54,5 \%)$. Estos datos de repuesta son comparables a otros trabajos que utilizaron metodologías similares (Hu, Primack, Barnett y Cook, 2011; Keeter, Miller, Kohut, Groves y Presser, 2000).

La muestra final fue de 37 médicos, mayoritariamente hombres $(n=20,54.1 \%)$; de los cuales 25 ejercían la especialidad de psiquiatría $(67.6 \%)$. La edad media de los encuestados fue de 40 años (DS 11.4). La experiencia media de todos los profesionales fue de 13.9 años ( $D S$ 11.2).

Los profesionales consideraron los riesgos asociados a la prescripción de benzodiacepinas moderadamente relevantes $(5.27 / 7)$, con fuerte evidencia científica $(5.59 / 7)$, con consecuencias a largo plazo $(5.27 / 7)$, largamente conocido $(2.35 / 7)$ y altamente controlable por el prescriptor $(5.27 / 7)$ pero poco familiar $(2.51 / 7)$, consentido $(3.06 / 7)$

Recibido: agosto 2013; Aceptado: noviembre 2013

Enviar correspondencia a:

Hugo López Pelayo. Hospital Clínic i Universitari de Barcelona. Servicio de Psiquiatría. Villarroel 170, escalera 9, planta 6, 08036, Barcelona, España. E-mail: hlopez@clinic.ub.es 
Tabla 1

Descripción de las dimensiones de la percepción de riesgo

\begin{tabular}{|c|c|c|c|}
\hline Dimensión & $\begin{array}{l}\text { Salud Mental }(n=12) \\
\text { Puntuación media (DS) }\end{array}$ & $\begin{array}{l}\text { Atención Primaria }(n=25) \\
\text { Puntuación media (DS) }\end{array}$ & $\begin{array}{c}\text { Global }(n=37) \\
\text { Puntuación media (DS) }\end{array}$ \\
\hline Importancia & $5.20(1.66)$ & $5.45(0.82)$ & $5.27(1.45)$ \\
\hline Inmediato/Largo plazo & $5.36(1.41)$ & $5.25(1.60)$ & $5.32(1.45)$ \\
\hline Conocimiento & $2.52(1.01)$ & $2.50(1.00)$ & $2.51(0.99)$ \\
\hline Consentimiento & $2.80(1.23)$ & $3.64(1.36)$ & $3.06(1.29)$ \\
\hline Evidencia & $5.68(1.70)$ & $5.42(1.44)$ & $5.59(1.60)$ \\
\hline Novedad & $2.16(1.21)$ & $2.75(1.36)$ & $2.35(1.27)$ \\
\hline Constancia & $4.60(1.26)$ & $5.33(0.79)$ & $4.84(1.17)$ \\
\hline Temor & $3.80(1.29)$ & $3.50(1.68)$ & $3.70(1.41)$ \\
\hline Gravedad & $4.60(1.80)$ & $4.82(0.75)$ & $4.66(1.55)$ \\
\hline Control -paciente- & $3.84(1.38)$ & $3.42(1.51)$ & $3.70(1.41)$ \\
\hline Control -prescriptor- & $5.40(1.35)$ & $5.00(1.48)$ & $5.27(1.39)$ \\
\hline Exposición -propios pacientes- & $4.32(1.57)$ & $4.67(1.72)$ & $4.43(1.61)$ \\
\hline Exposición -pacientes salud mental- & $4.92(1.19)$ & $5.83(0.84)$ & $5.22(1.16)$ \\
\hline Exposición-pacientes primaria- & $4.36(1.47)$ & $4.92(1.17)$ & $4.54(1.39)$ \\
\hline Observabilidad & $3.92(1.53)$ & $4.00(1.28)$ & $3.95(1.43)$ \\
\hline Cambio en el riesgo & $3.76(1.72)$ & $3.92(1.78)$ & $3.81(1.71)$ \\
\hline Dificultad de reducción & $3.25(1.36)$ & $4.75(1.36)$ & $3.75(1.52)$ \\
\hline Uso en ancianos & $5.41(1.33)$ & $6.08(0.90)$ & $5.65(1.23)$ \\
\hline Uso en niños y adolescentes & $4.23(1.51)$ & $6.25(1.42)$ & $4.94(1.76)$ \\
\hline Uso en embarazadas & $4.62(1.56)$ & $5.42(1.73)$ & $4.91(1.64)$ \\
\hline Uso en enfermos neurológicos (sistema nervioso central) & $4.76(1.34)$ & $4.92(1.38)$ & $4.82(1.33)$ \\
\hline Uso en pacientes con enfermedad mental & $4.41(1.40)$ & $5.00(0.85)$ & $4.62(1.26)$ \\
\hline Uso en pacientes con trastorno uso sustancia activo & $6.68(0.48)$ & $6.00(1.13)$ & $6.44(0.82)$ \\
\hline Uso en pacientes con trastorno uso sustancia en el pasado & $6.23(0.87)$ & $5.33(1.23)$ & $5.91(1.08)$ \\
\hline Uso en pacientes con rastorno mental severo & $4.41(1.62)$ & $4.75(1.36)$ & $4.53(1.52)$ \\
\hline Uso en pacientes con trastorno de la personalidad & $5.50(1.30)$ & $5.25(1.14)$ & $5.41(1.23)$ \\
\hline
\end{tabular}

y no fácil de controlar por el paciente (3.70/7). El resto de dimensiones son expuestas en la tabla 1.

Comparado con los psiquiatras, los profesionales de AP consideraron el riesgo asociado a las benzodiacepinas de más difícil reducción $(\mathrm{p}=.005)$ y que afecta a un gran número de pacientes $(\mathrm{p}=.037)$. Los pacientes de salud mental $(\mathrm{p}=.011)$ y los niños y adolescentes $(\mathrm{p}=.01)$ fueron vistos como los grupos más expuestos. En contraste, los profesionales de SM percibieron un alto riesgo $(\mathrm{p}=.039)$ en relación con el abuso en el pasado de sustancias.

En conclusión, incluso con importantes diferencias en dimensiones específicas entre profesionales de SM y AP, la mayoría de profesionales tienen una elevada percepción riesgo. Los profesionales están de acuerdo en que hay una mala percepción por parte del paciente. Se abre una puerta a intervenciones en pacientes de atención primaria para incrementar la percepción de riesgo ya que hasta un $5 \%$ de las consultas en este ámbito concluyen con la prescripción de benzodiazepinas (Berjano et al., 2008). Se debería prestar atención a los grupos poblacionales de riesgo (Billioti de Gage et al., 2012; Formiga et al., 2012).

\section{Reconocimientos}

Agradecemos la participación de los diversos profesionales de salud mental y atención primaria que han atendido nuestra petición de estudio. También queremos reconocer la colaboración prestadas por las entidades sanitarias (Hospital Clínic i Universitari de Barcelona, Centro de Salud Mental Esquerra de l'Eixample, Unidad de Conductas Adictivas del Hospital Clínic i Universitari, Centro de Atención Primaria Roselló) y los órganos de coordinación que han facilitado este trabajo. 


\section{Conflicto de intereses}

Los firmantes declaran no tener conflictos de intereses.

\section{Referencias}

Agencia Española del Medicamento (2000). Circular 3/2000: Información a incluir en las fichas técnicas de especialidades farmacéuticas que contengan benzodiacepinas. Madrid: Ministerio de Sanidad y Consumo. Recuperado de: http://www.aemps.gob.es/informa/circulares/medicamentosUsoHumano/2000/

Berjano Romero, F., Piñol Moreso, J. L., Mora Gilabert, N., Claver Luque, P., Brull López N. y Basora Gallisà, J. (2008). Elevado consumo de benzodiacepinas en mujeres ancianas asignadas a centros de salud urbanos de atención primaria. Atención Primaria, 40, 617-622. doi: 10.4321/S1699-695X2011000200002

Billioti de Gage, S., Bégaud, B., Bazin, F., Verdoux, H., Dartigues, J. F., Pérès, K.,... Pariente, A. (2012). Benzodiazepine use and risk of dementia: prospective population based study. British Medical Journal, 345:e6231. doi: 10.1136/bmj.e6231

Formiga, F., Duaso, E., Ruiz, D., San José, A., Urrutia, A. y López-Soto, A. (2012). Utilización de benzodiacepinas según el motivo de ingreso. Medicina Clinica (Barc), 139, 531-534. doi: 10.1016/j.medcli.2012.05.037

García del Pozo, J., Abajo Iglesias, F. J., Carvajal García-Pando, A., Montero Corominas, D., Madurga Sanz, M. y García del Pozo, V. (2004). Utilización de ansiolíticos e hipnóticos en España (1995-2002). Revista Española de Salud Publica, 78, 379-387. doi: 10.1590/S113557272004000300007

Hu, X., Primack, B. A., Barnett, T. E. y Cook, R. L. (2011). College students and use of K2: an emerging drug of abuse in young persons. Substance Abuse: Treatment, Prevention and Policy, 11, 6-16. doi: 10.1186/1747-597X-6-16.

Keeter, S., Miller, C., Kohut, A., Groves, R. M. y Presser, S. (2000). Consequences of reducing nonresponse in a national telephone survey. Public Opinion Quarterly, 64, 125-148. doi: 10.1086/317759

Plan Nacional sobre Drogas. Ministerio de Sanidad y Consumo (2009). Encuesta domiciliaria sobre alcohol y drogas en España 2009 (EDADES 2009). Madrid: Ministerio de Sanidad y Consumo. Recuperado de http://www.pnsd.msc. es/Categoria2/observa/pdf/EDADES_2009.pdf

Puy Rodríguez, A. (1994). Percepción social del riesgo. Dimensiones de evaluación y predicción. Tesis doctoral en psicología social. Madrid: Universidad Complutense de Madrid.

Slovic, P. (1987). Perception of risk. Science, 236, 280-285. doi: $10.1126 /$ science. 3563507 